Although there is a superficial resemblance between the cratering of Mars and that of the Moon, in many cases the morphology of the Martian craters is quite different from that of lunar craters. The dark spots known as Nix Olympica, North Spot and South Spot are particularly interesting. Each spot contains a crater or crater complex, the Nix Olympica and North Spot four or five overlapping craters each. These seem to have formed successively, have very low rims, and do not have surrounding sharp ridges like those of most lunar craters. South Spot is a single structure roughly $100 \mathrm{~km}$ across, and unlike any known terrestrial or lunar crater. Several small rimless craters are seen on its rim, and some of these form slightly bent chains. Major valleys extend to the north-east and south-west of the South Spot, and it may not be a coincidence that this is also the direction of alignment of the North Spot, Middle Spot and South Spot.

In all of these spots there is morphological evidence of subsidence, and the broad features are reminiscent of volcanic calderas. It is difficult to explain their appearance as the result of meteoritic impacts. Also, the sharpness of the features and the smoothness of the surrounding terrain suggest that the spots are geologically young features, contrasting with the heavily cratered areas observed by earlier Mariner probes. The striking pictures of Phobos and Deimos also obtained by Mariner 9 provide a control by which the effects of weathering on Martian craters can be estimated, and these confirm that Mars is very much an active planet.

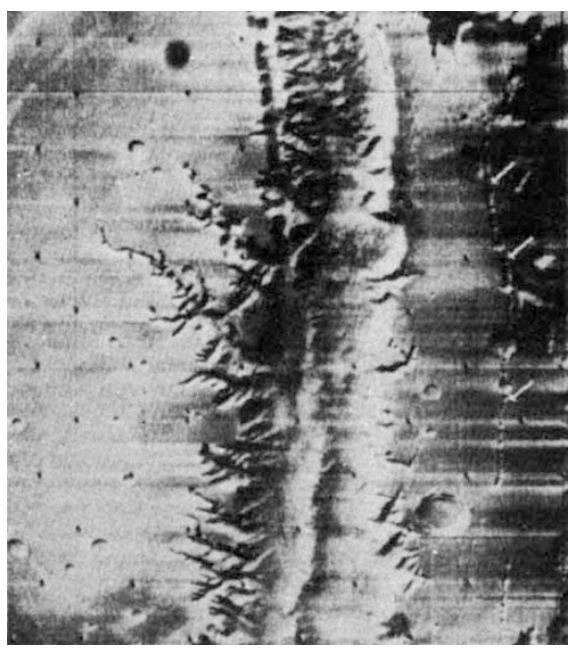

Fig. 2 Erosion pattern in the walls of a 50 mile wide down-faulted valley on Mars. Subsidence caused by volcanism and wind erosion might have caused the weathered appearance, which resembles scouring patterns produced by occasional heavy rain in arid regions of Earth. Arrows mark a crater chain like those found on the Moon. This may be the result of venting through a fracture in the crust.

VISION

\section{Dark Happenings}

from our Molecular Biology Correspondent

Aside from recent alarums and excursions, which are now known to have been fruitless, concerning a supposed covalent association of retinal with lipids, the train of chemical events in the bleaching of rhodopsin has stood solidly established for some time. The full horse-power of effort and conjecture has centred on the mechanism of excitation that follows this process. The question of regeneration of the rhodopsin in preparation for the arrival of the next quantum has by contrast received much less attention. Now, however, some attributes of this phase of the visual cycle have been uncovered.

In the first place, Shichi (J. Biol. Chem., 246, 6178; 1971) has confirmed that rhodopsin requires at least some of its bound lipid to function in this respect. The protein resides, of course, in the membranes stacked in the rod outer segment, and as extracted in its functional state remains associated with a substantial amount of lipid. In intact rod outer segments, the ability of the pigment to regenerate is impaired by solvent extraction of lipids, and annihilated by treatment with phospholipase. If a solvent extract of lipid is restored to the depleted rod, the capacity to regenerate is regained. Moreover, single phospholipid components out of bottles serve just as well, but neutral lipids are ineffective. A simple interpretation of these effects is that the anionic phospholipids bind to the opsin, and exert a conformationally stabilizing effect (just as, for example, the native conformation of serum lipoproteins is disturbed by removal of lipid and restored when endogenous or extraneous lipids are added back).

Such an explanation is evidently on the right lines, for either phospholipase

\title{
Carbon Chemistry of the Lunar Surface
}

THE search for complex organic molecules in samples of the lunar surface brought back by the Apollo. landings has given way to a study of the traces of light hydrocarbons and carbides which seem to be indigenous to this material. The low levels of total carbon (10-200 p.p.m.) and carbon compounds such as methane (0.1-5.0 p.p.m.) found in these samples require the development by organic geochemists of refined techniques for the handling and analysis of lunar material. Two of the university groups engaged in these lunar carbon studies are the Organic Geochemistry Unit at Bristol and the Space Sciences Laboratory at Berkeley, California, and in next Monday's Nature Physical Science (February 7) there are complementary contributions from these two laboratories.

Previous work by Eglinton et al. (Nature, 231, 29; 1971) on Apollo 11 and 12 lunar fines suggested that traces of indigenous methane were probably derived chiefly from solar wind implantation, with minor contributions from lunar primordial sources. Lunar carbides or "carbide-like" material thus seemed to be of extra-lunar origin with contributions from both the solar wind and debris from meteoritic impact, the second probably being of lesser importance. The Bristol group now report the study of the simulation of solar wind bombardment of various lunar samples and metallic surfaces.

Targets of the various materials were irradiated in an isotope separator by ionized pieces of carbon $\left({ }^{13} \mathrm{C}^{+}\right)$and deuterium $\left(\mathrm{D}_{2}{ }^{+}\right)$at energies similar to those estimated for the solar wind. The isotopically labelled species released by acid treatment or pyrolysis of the implanted targets were then examined by mass spectrometry. Methane from the irradiated lunar fines was shown to be synthesized from the bombarding ions, and material reacting as carbide was synthesized in the metal targets. These simulation experiments also indicated that the meteoritic contribution to lunar "carbides" may be greater than previously suggested.

The studies of the Berkeley group are of particular interest because they include an examination of an Apollo 14 sample taken in the Special Environmental Sample container which was sealed on the Moon and not opened until inside the University of California's ultra clean handling facility. Gases released from this and other Apollo 14 samples by deuteriumlabelled acid etching treatment showed that the amount of methane and neon and argon derived from the solar wind were related to grain surface area, confirming previous results for Apollo 11 and 12 fines. The pattern of evolved deuterium labelled hydrocarbons was similar to that produced from carbides, and grains of carbides have now been identified in lunar fines. This observation, taken in conjunction with calculations of the likely amount of carbon from solar wind bombardment, again suggests an important contribution from meteoritic material, supporting the suggestions of the Bristol group. The high degree of ${ }^{13} \mathrm{C}$-isotopic enrichment observed in lunar fines might therefore result from fractionation during vaporization and deposition of meteoritic carbide, as well as from the "hydrogen stripping" process. 